\title{
Shannon Entropy Density as a Descriptor of Coulomb Systems
}

\author{
Á. Nagy \\ Department of Theoretical Physics, \\ University of Debrecen, \\ H-4010 Debrecen, Hungary
}

(Dated: November 21, 2012)

\begin{abstract}
It is shown that the Shannon entropy density determines every property of a finite Coulomb system both in the ground and the excited states. Cusp relations and the asymptotic behaviour for the Shannon entropy density are presented.
\end{abstract}




\section{INTRODUCTION}

It is well-known that the ground-state electron density is a fundamental quantity as it determines every property of the electron system [1]. It has recently turned out, however, that there exist other descriptors of a Coulomb system. Ayers [2] proved that the shape function, (that is, the density per particle) provides a full description of a Coulomb system. Reactivity indicators [3] as Fukui function [4], local softness, softness kernel and the electrostatic potential are also contain all information about a Coulomb system. It has also turned out [5] that local kinetic energy and local temperature are also adequate for describing a Coulomb system. Moreover, an even broader family of descriptors of Coulomb systems (certain linear combinations of the one-particle Kohn-Sham densities) were presented [6] .

The descriptors mentioned above contain all information about a Coulomb system. Nowadays information theoretical concepts received considerable attention. One might ask whether Shannon information entropy density [7] provides a full description of a Coulomb system. The answer is the affirmative. In this paper it is proved that the Shannon information entropy density determines every property of a finite Coulomb system.

The Hamiltonian of a Coulomb system can be written as

$$
\begin{gathered}
\hat{H}=\hat{T}+\hat{V}+\hat{V}_{e e} . \\
\hat{T}=\sum_{j=1}^{N}\left(-\frac{1}{2} \nabla_{j}^{2}\right), \\
\hat{V}_{e e}=\sum_{k=1}^{N-1} \sum_{j=k+1}^{N} \frac{1}{\left|\mathbf{r}_{k}-\mathbf{r}_{j}\right|},
\end{gathered}
$$

and

$$
\hat{V}=\sum_{k=1}^{N} \sum_{\alpha=1}^{M} \frac{-Z_{\alpha}}{\left|\mathbf{r}_{k}-\mathbf{R}_{\alpha}\right|},
$$

are the kinetic energy, the electron-electron energy and the electron-nucleon operators, respectively. If we know the atomic numbers $Z_{\alpha}$, the position of the nuclei $\mathbf{R}_{\alpha}(\alpha=1, \ldots, M$ ) and the number of electrons $N$, the Hamiltonian is also known and solving the eigenvalue problem

$$
\hat{H} \Psi_{k}=E_{k} \Psi_{k} \quad(k=1, \ldots, i, \ldots)
$$


any property of the system can in principle be obtained.

The fact that the ground-state electron density is sufficient in principle to determine all molecular properties, can be simply understood following Bright Wilson's [8,9] argument: A well-known theorem of quantum mechanics, Kato's theorem [10-12] states that

$$
Z_{\alpha}=-\left.\frac{1}{2 n(r)} \frac{\partial \bar{n}(r)}{\partial r}\right|_{r=R_{\alpha}},
$$

where the partial derivatives are taken at the nuclei $\alpha$ and $\bar{n}(r)$ is the angular average of the density. So the cusps of the density tell us where the nuclei are $\left(R_{\alpha}\right)$ and what the atomic numbers $Z_{\alpha}$ are. On the other hand, the integral of the density gives us the number of electrons:

$$
N=\int n(\mathbf{r}) d \mathbf{r}
$$

Thus from the density the Hamiltonian can be readily obtained from which every property can be determined.

The Shannon entropy density can be easily calculated from the electron density $n(\mathbf{r})$

$$
s(\mathbf{r})=-n(\mathbf{r}) \ln n(\mathbf{r})
$$

The Shannon information entropy [7] is given by $S=\int s(\mathbf{r}) d \mathbf{r}$.

At first sight one might think that the density $n$ can be expressed from Eq. (8) as a function of $s$. Using the Lambert function [13] $W(x)$ with the definition

$$
x=W(x) e^{W(x)}
$$

the density can be written as

$$
n=e^{W(-s)} .
$$

However, $W(x)$ is a multivalued function. For real $x$ it has two branches $W_{-1}(x)$ and $W_{0}(x)$ as it represented in Fig. 1. $W_{-1}(x)$ is defined for $-1 / e \leq x<0 . W_{0}(x)$ is defined for $-1 / e \leq x<+\infty$. Fig. 2. and 3. present the Hartree-fock density as a function of $-s$ for the $\mathrm{H}$ and $\mathrm{Ne}$ atoms. In case of the $\mathrm{H}$ atom only the branch $W_{-1}$ appears. For other atoms, such as the Kr atom (Fig. 3) both branches are realized. So, the density cannot generally uniquely obtained from Eq. (10). Therefore, to prove that the Shannon entropy density is a descriptor of a Coulomb system, the cusp relations and the asymptotic behaviour should be studied. 


\section{CUSP RELATIONS AND ASYMPTOTIC BEHAVIOUR FOR THE SHANNON ENTROPY DENSITY}

Cusp relations on the wave function and the electron density have been studied by several authors $[10,11,14-19]$. The most general expansion of the antisymmetric wave function around the nucleus $\alpha$ is

$$
\Phi=\sum_{l=0}^{\infty} \sum_{m=-l}^{m=l} r_{1}^{l} \chi_{l m}\left(r_{1}, X\right) Y_{l m}\left(\Omega_{1}\right),
$$

where $X$ stands for the coordinates $\mathbf{r}_{2}, \ldots, \mathbf{r}_{N} . Y_{l m}$ are the spherical harmonics. (In certain highly excited states the spherical average of the derivative of the wave function is zero at a nucleus: $\chi_{00}(0, X)=0$. This case were studied in Ref. [19]. Here only ground or low-lying excited states are considered.) The function $\chi_{l m}$ can be expanded as

$$
\chi_{l m}(r, X)=a_{l m}^{(0)}(X)+a_{l m}^{(1)}(X) r+a_{l m}^{(2)}(X) r^{2}+a_{l m}^{(3)}(X) r^{3}+\ldots
$$

The derivative of the spherical average of the Shannon entropy density (Eq. (8))

$$
\frac{\partial \bar{s}}{\partial r}=-\frac{1}{4 \pi} \int \frac{\partial n(r, \Omega)}{\partial r}(1+\ln n(r, \Omega)) d \Omega .
$$

Following the steps in Ref. [19] one can readily obtain that at the nucleus $\alpha$

$$
\left.\frac{\partial \bar{s}(r)}{\partial r}\right|_{\mathbf{r}=\mathbf{R}_{\alpha}}=2 Z_{\alpha} n\left(\mathbf{R}_{\alpha}\right)\left(1+\ln n\left(\mathbf{R}_{\alpha}\right)\right) .
$$

Taking into account that the Shannon entropy density at the nucleus $\alpha$ is

$$
s\left(\mathbf{R}_{\alpha}\right)=-n\left(\mathbf{R}_{\alpha}\right) \ln n\left(\mathbf{R}_{\alpha}\right)
$$

we arrive at the cusp relation

$$
\frac{\left.\frac{\partial \bar{s}(r)}{\partial r}\right|_{\mathbf{r}=\mathbf{R}_{\alpha}}}{s\left(\mathbf{R}_{\alpha}\right)}=-2 Z_{\alpha} \frac{\left(1+\ln n\left(\mathbf{R}_{\alpha}\right)\right)}{\ln n\left(\mathbf{R}_{\alpha}\right)} .
$$

It can also be written as

$$
\frac{\left.\frac{\partial \bar{s}(r)}{\partial r}\right|_{\mathbf{r}=\mathbf{R}_{\alpha}}}{s\left(\mathbf{R}_{\alpha}\right)}=-2 Z_{\alpha} \frac{\left(s\left(\mathbf{R}_{\alpha}\right)-n\left(\mathbf{R}_{\alpha}\right)\right.}{s\left(\mathbf{R}_{\alpha}\right)} .
$$

From the cusp condition (17) the atomic number can be obtained as

$$
Z_{\alpha}=\frac{1}{2} \frac{\left.\frac{\partial \bar{s}(r)}{\partial r}\right|_{\mathbf{r}=\mathbf{R}_{\alpha}}}{n\left(\mathbf{R}_{\alpha}\right)-s\left(\mathbf{R}_{\alpha}\right)}
$$




$$
Z_{\alpha}=\frac{1}{2} \frac{\left.\frac{\partial \bar{s}(r)}{\partial r}\right|_{\mathbf{r}=\mathbf{R}_{\alpha}}}{n\left(\mathbf{R}_{\alpha}\right)\left[1+\ln \left(n\left(\mathbf{R}_{\alpha}\right)\right)\right]} .
$$

Consequently, $Z_{\alpha}$ can be determined from $s$ if $n\left(\mathbf{R}_{\alpha}\right)$ is known. $n\left(\mathbf{R}_{\alpha}\right)$ can be obtained from Eq. (10). Though Eq. (10) provides two solutions, only one of them gives a positive atomic number. The density $n\left(\mathbf{R}_{\alpha}\right)$ should be positive, therefore, if $\partial \bar{s}(r) /\left.\partial r\right|_{r=R_{\alpha}}>0$ we get $n>1 / e$, that is, $n\left(\mathbf{R}_{\alpha}\right)=\exp \left(W_{0}\left(-s\left(\mathbf{R}_{\alpha}\right)\right)\right.$. On the other hand, $\partial \bar{s}(r) /\left.\partial r\right|_{r=R_{\alpha}}<0$ leads to $n<1 / e$, that is, $n\left(\mathbf{R}_{\alpha}\right)=\exp \left(W_{-1}\left(-s\left(\mathbf{R}_{\alpha}\right)\right)\right.$. Finally, if $\partial \bar{s}(r) /\left.\partial r\right|_{r=R_{\alpha}}=0$, we cannot use Eq. (19) to obtain the atomic number as $1+\ln n\left(\mathbf{R}_{\alpha}\right)=0$. In this case the second derivative of $s$ should be considered. We mention in passing that a similar situation can be found for the density in highly excited states, where the first derivative of the density disappears [19].

If $1+\ln n\left(\mathbf{R}_{\alpha}\right)=0$, the second derivative of the Shannon entropy density at the the nucleus $\alpha$ is given by

$$
\left.\frac{\partial^{2} \bar{s}(r)}{\partial r^{2}}\right|_{\mathbf{r}=\mathbf{R}_{\alpha}}=-\left.\frac{1}{n\left(\mathbf{R}_{\alpha}\right)}\left(\frac{\partial \bar{n}(r)}{\partial r}\right)^{2}\right|_{\mathbf{r}=\mathbf{R}_{\alpha}} .
$$

Taking into account the cusp relation (6) for the density we arrive at

$$
\left.\frac{\partial^{2} \bar{s}(r)}{\partial r^{2}}\right|_{\mathbf{r}=\mathbf{R}_{\alpha}}=-4 Z_{\alpha}^{2} n\left(\mathbf{R}_{\alpha}\right)=-4 Z_{\alpha}^{2} s\left(\mathbf{R}_{\alpha}\right) .
$$

The last step follows from the fact that if $1+\ln n\left(\mathbf{R}_{\alpha}\right)=0$, Eq. (8) gives $s\left(\mathbf{R}_{\alpha}\right)=n\left(\mathbf{R}_{\alpha}\right)$. Consequently, the cusp relation takes the form

$$
\frac{\left.\frac{\partial^{2} \bar{s}(r)}{\partial r^{2}}\right|_{\mathbf{r}=\mathbf{R}_{\alpha}}}{s\left(\mathbf{R}_{\alpha}\right)}=-4 Z_{\alpha}^{2}
$$

From the cusp condition (22) the atomic number can be obtained as

$$
Z_{\alpha}=\frac{1}{2}\left(-\frac{\left.\frac{\partial^{2} \bar{s}(r)}{\partial r^{2}}\right|_{\mathbf{r}=\mathbf{R}_{\alpha}}}{s\left(\mathbf{R}_{\alpha}\right)}\right)^{1 / 2}
$$

These results can be summarized in

THEOREM 1. For any Coulomb system, the Shannon entropy density $s$ determines the external potential $v$, up to an additive constant. 
Proof: The external potential of a Coulomb system has the form

$$
v(\mathbf{r})=\sum_{\alpha=1}^{M} \frac{-Z_{\alpha}}{\left|\mathbf{r}-\mathbf{R}_{\alpha}\right|} .
$$

The cusps in $s$ determine the positions $\mathbf{R}_{\alpha}$ of the nuclei and the atomic numbers $Z_{\alpha}$ (Eq. (19)). In case $\partial \bar{s} /\left.\partial r\right|_{\mathbf{R}_{\alpha}}=0$, the atomic numbers $Z_{\alpha}$ are given by Eq.(23).

To prove that the Shannon entropy density $s$ also determines the number of electrons $N$ the asymptotic behaviour of $s$ should be studied.

It is well-known [20-25] that the asymptotic behaviour of the density $n$ is

$$
n(r) \rightarrow r^{2 \beta} e^{-2 \kappa r},
$$

where

$$
\begin{gathered}
\beta=\frac{Z_{\text {total }}-N+1}{\kappa}-1 . \\
Z_{\text {total }}=\sum_{\alpha=1}^{M} Z_{\alpha}
\end{gathered}
$$

and

$$
\kappa=\sqrt{2 I}
$$

$I$ is the ionization potential. Using Eqs. (8) and (25) we are led to

$$
\lim _{r \rightarrow \infty} \frac{\partial \ln s}{\partial r}=\lim _{r \rightarrow \infty} \frac{\partial \ln n}{\partial r}=-2 \kappa=-2 \sqrt{2 I} .
$$

Combining Eqs. (8), (25) and (29) we obtain

$$
N=1+Z_{\text {total }}+\frac{1}{4} \lim _{r \rightarrow \infty}\left[\frac{\partial \ln s}{\partial r}\left(1+\frac{\partial\left(\frac{\partial \ln s}{\partial r}\right)}{\partial\left(\frac{1}{r}\right)}\right)\right] .
$$

Thus we proved

THEOREM 2. For the ground state of any Coulomb system, the Shannon entropy density $s$ determines the number of electrons bound to the system, $N$.

Theorem 2 implies that in a Coulomb system $N$ is a functional of $s$.

THEOREM 3. For the ground state of any Coulomb system, the knowledge of the Shannon entropy density $s$ is sufficient for obtaining the value of any observable. 
Proof: According to Theorems 1 and $2 s$ determines the external potential $v$ and the number of electrons $N$. From these quantities the Hamiltonian can be constructed. The Hamiltonian gives the wave function, which deternmines the value of any observable.

Theorems 1-3 are valid to excited states, too.

THEOREM 4. For any Coulomb system, the knowledge of the Shannon entropy density $s$ is sufficient for obtaining the value of any observable.

Proof: Both Theorems 1 and 2 are valid both for the ground and the excited states. $s$ determines $v$ and $N$ and hence $\hat{H}$. To find out which eigenstate of $\hat{H}$ corresponds to $s$, one has to find first the energy. The energy $E_{k}$ of the excited state can be obtained by

$$
\lim _{r \rightarrow \infty} \frac{\partial \ln s}{\partial r}=-2 \sqrt{2 I_{k}}
$$

where

$$
I_{k}=E_{k}-E_{1}^{(N-1)}
$$

$E_{1}^{(N-1)}$ is the ground-state energy of the system after an electron is removed. From the knowledge of the ground-state energy of the $N-1$ electron system Eqs. (31) and (32) give the energy corresponding to $s$. Then we can find the eigenstate having energy $E_{k}$ and Shannon entropy density $s$.

\section{DISCUSSION}

The conclusion of this paper is that the Shannon entropy density $s$ contains all information about a Coulomb system. It is important to emphasize that this finding is restricted to Coulomb system. We have utilized that the form of the Hamiltonian is given (by Eq. (1)(4) and the parameters (in $\hat{H}$ ) are determined by the cusp and the asymptotic behaviour of the Shannon entropy density $s$.

The theorems above imply that any observable property is a functional of $s$. For example the total energy can also be considered as a functional of $s$. The exact form of the total energy as a functional of the density $n$ is unkown. or rather, the kinetic and the correlation energy functionals are unkown. It might turn out that these energy functionals can be more easily approximated as functionals of $s$ than as $n$. There have already been such kind of attempts. Collins [26] conjectured that the correlation energy is proportional to the Shannon 
entropy [7]. Numerical results supported this conjecture for small molecules [27]. Collins' conjecture was modified for the He isoelectronic series [28]. Several papers studied the link between the correlation energy and Shannon information (e. g. [29-35]). Delle Site and collaborators [36, 37] proposed a kinetic energy functional in which a term is proportional to the Shannon entropy. The entropy density is of significant interest in the recently trendy Hirshfeld-like partitioning methods [38-41].

In a recent paper [42] a theory of excited states has been put forward for Coulomb system. In this paper functionals have been defined for all densities not only for Coulomb densites. A similar construction can be done for the functionals of $s$, too. Then the variational principle can be also formalized. Moreover, it will be possible to formulate a bifunctional theory (similar to the Levy-Nagy theory $[43,44]$ or the generalized density functional theory [4549]), where the functionals will depend on both $s$ and $n$. It will be the subject of future research.

[1] P. Hohenberg and W. Kohn, Phys. Rev. 136 (1864) B864.

[2] P. W. Ayers, Proc. Natl. Acad. Sci.97 (2000) 1959.

[3] P. W. Ayers, Chem. Phys. Lett.438 (2007) 148.

[4] F. De Proft, P. W. Ayers, K. D. Sen, and P. Geerlings, J. Chem. Phys. 120(2004) 9969.

[5] P. W. Ayers and Á. Nagy, J. Chem. Phys. 126 (2007) 144108.

[6] Á. Nagy, Chem. Phys. Lett.460(2008) 343.

[7] C. E. Shannon, Bell Syst. Tech. J. 27(1948) 379.

[8] N. C. Handy, in Quantum Mechanical Simulation Methods for Studying Biological Systems Eds. D. Bicout and M. Field (Springer-Verlag, Heidelberg,1996) p.1.

[9] Á. Nagy, Int. J. Quantum Chem. 70 (1998) 681.

[10] T. Kato, Commun. Pure Appl. Math. 10(1957) 151.

[11] E. Steiner, J. Chem. Phys. 39(1963) 2365.

[12] N. H. March, Self-consistent fields in atoms (Pergamon, Oxford, 1975).

[13] see e. g. F. Chapeau-Blondeau, IEEE Trans. Sig. Proc. 50 (2002) 2160.

[14] R. T. Pack and W. B. Brown, J. Chem. Phys. 45 (1966) 556.

[15] R. O. Esquivel, J. Chen, M. J. Stott, R. P. Sagar and V. H. Smith, Jr., Phys. Rev. A 47(1993) 
936.

[16] R. O. Esquivel, R. P. Sagar, V. H. Smith, Jr., J. Chen and M. J. Stott, Phys. Rev. A 47(1993) 4735 .

[17] Á Nagy and K. D. Sen, J. Phys. B 33(2000) 1745.

[18] Á Nagy and K. D. Sen, Chem. Phys. Lett. 332, 154 (2000).

[19] Á Nagy and K. D. Sen, J. Chem. Phys. 115, 6300 (2001).

[20] M. M. Morrell, R. G. Parr and M. Levy, J. Chem. Phys. 62(1975) 549.

[21] R. Ahlrichs, J. Chem. Phys. 64(1976) 2706.

[22] M. Hoffmann-Ostenhof and T. Hoffmann-Ostenhof, Phys. Rev. A 16(1977)1782 .

[23] H. J. Silverstone, Phys. Rev. A 23(1981) 1030.

[24] M. Levy, J. P. Perdew and V. Sahni, Phys. Rev. A 30(1984)2745 .

[25] C.-O. Almbladh and U. von Barth, Phys. Rev. B 31(1985)3231.

[26] D. M. Collins, Z. Naturforsch,48(1993) 68.

[27] J. C. Ramírez, C. Soriano, R. O. Esquivel, R. P. Sagar, M. Ho and V. H. Smith, Jr, Phys. Rev. A 56(1997) 4477.

[28] P. Ziesche, V. H. Smith, M. Ho, P. Gersdorf and M. Taut, J. Chem. Phys. 110(1999) 6135.

[29] P. Ziesche, Int. J. Quantum. Chem. 56 (1995)363.

[30] R. O. Esquivel, A. L. Rodríguez, R. P. Sagar, M. Ho and V. H. Smith, Jr, Phys. Rev. A 54(1996) 259.

[31] G. T. Smith, H. L. Schmider and V. H. Smith, Jr, Phys. Rev. A 65(2002) 032508.

[32] P. Gersdorf, W. John, J. P. Perdew and P. Ziesche, Int. J. Quantum. Chem. 61(1998)935.

[33] P. Ziesche, Theochem, 527(2000)35.

[34] N. L. Guevara, R. P. Sagar and R. O. Esquivel, J. Chem. Phys. 122(2005) 084101.

[35] N. L. Guevara, R. P. Sagar and R. O. Esquivel, Phys. Rev. A 67(2003)012507.

[36] L. Delle Site, Eur. Phys. Lett. 86(2009) 40004.

[37] I. M. Ghiringhelli, L. Delle Site, R. A. Mosna and I. P. Hamilton, J. Math. Chem. 48 (2010)78.

[38] R. F. Nalewajski and R. G. Parr, Proc. Natl. Acad. Sci. 97(2000)8879.

[39] P. W. Ayers, J. Chem. Phys. 113(2000) 10886.

[40] R. G. Parr, P. W. Ayers, and R. F. Nalewajski, J. Phys. Chem. A 109 (2005) 3957.

[41] P. Bultinck, C. Van Alsenoy, P. W. Ayers, and R. Carb-Dorca, J. Chem. Phys. 126(2007) 144111. 
[42] P. Ayers, M. Levy and Á. Nagy, Phys. Rev. A 85(2012) 042518.

[43] M. Levy and Á. Nagy, Phys. Rev. Lett. 83(1999)4361.

[44] Á. Nagy and M. Levy, Phys. Rev. A 63(2001)2502.

[45] Á. Nagy, S. B. Liu and L. Bartolotti, J. Chem. Phys. 122(2005) 134107.

[46] M. Higuchi and K. Higuchi, Phys. Rev. B 69(2004)035113.

[47] M. Higuchi and K. Higuchi, Phys. Rev. B 69(2004) 165118.

[48] M. Higuchi and K. Higuchi, J. Magn. and Magn. Materials 272-76 (2004)659.

[49] P. W. Ayers and P. Fuentealba, Phys. Rev. A 80(2009) 032510.

\section{Acknowledgments}

The work is also supported by the TAMOP 4.2.1/B-09/1/KONV-2010-0007 and the TAMOP 4.2.2/B-10/1-2010-0024 projects. The project is co-financed by the European Union and the European Social Fund. Grant OTKA No. K 100590 is also gratefully acknowledged. 


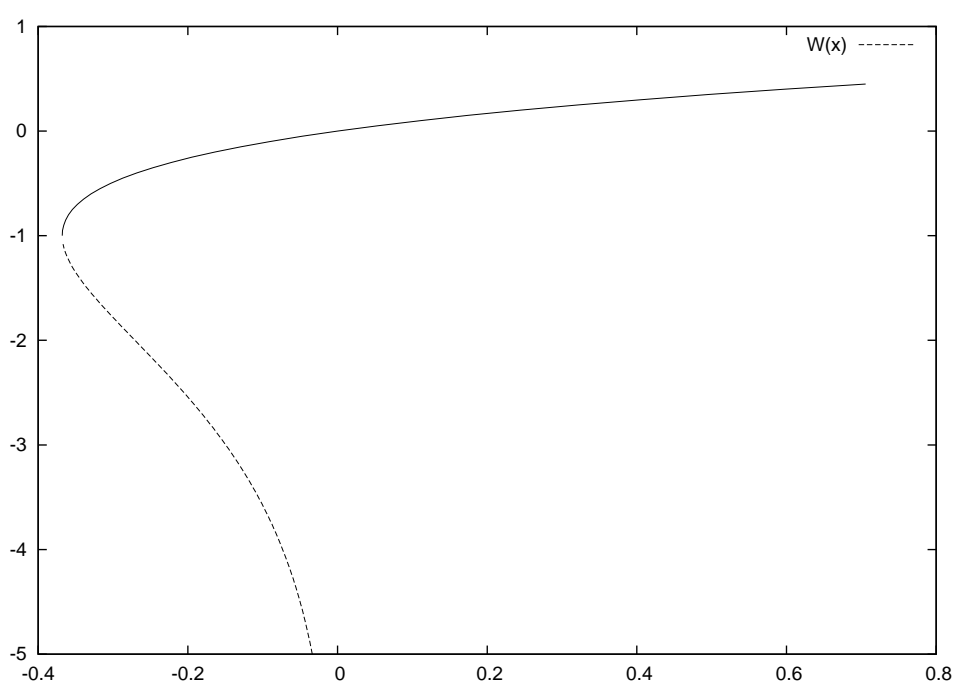

FIG. 1: Lambert function $W(x) . W_{-1}(x)$ is defined for $-1 / e \leq x<0 . W_{0}(x)$ is defined for $-1 / e \leq x<+\infty$. The two branches meet at the point $(-1 / e,-1)$.

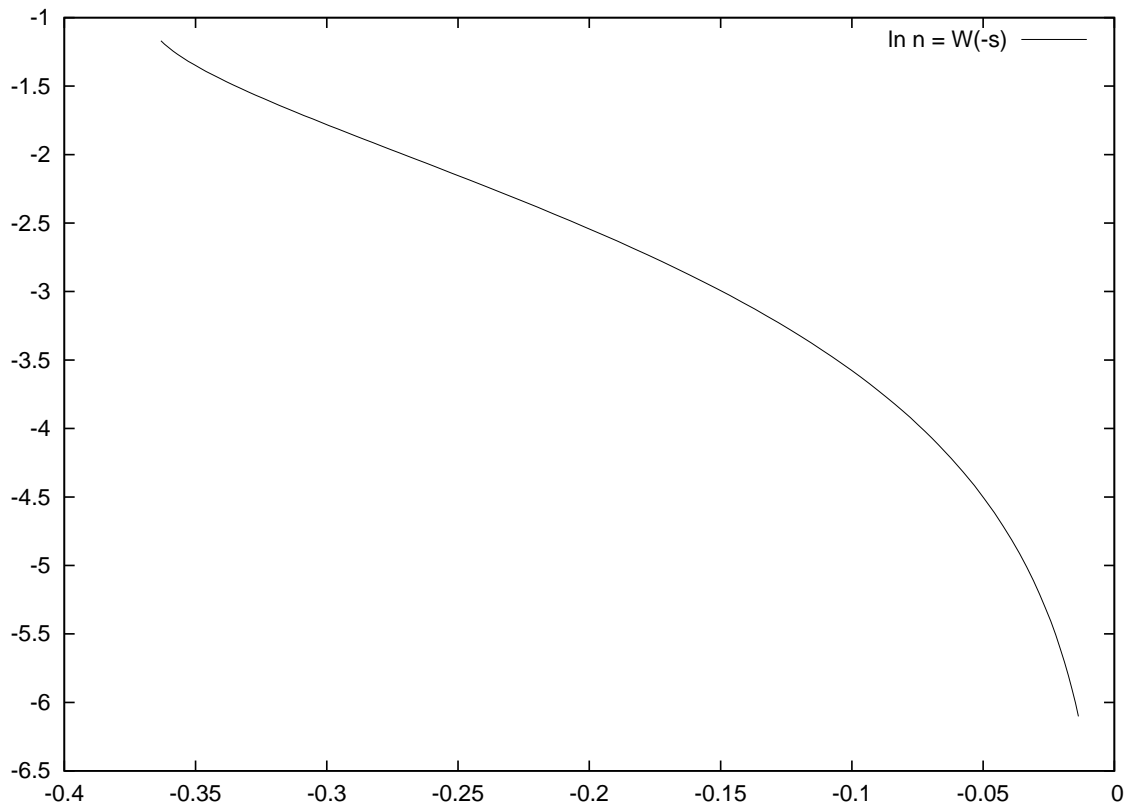

FIG. 2: The logarithm of the density of the Hydrogen atom as a function of the negative of the local entropy. 


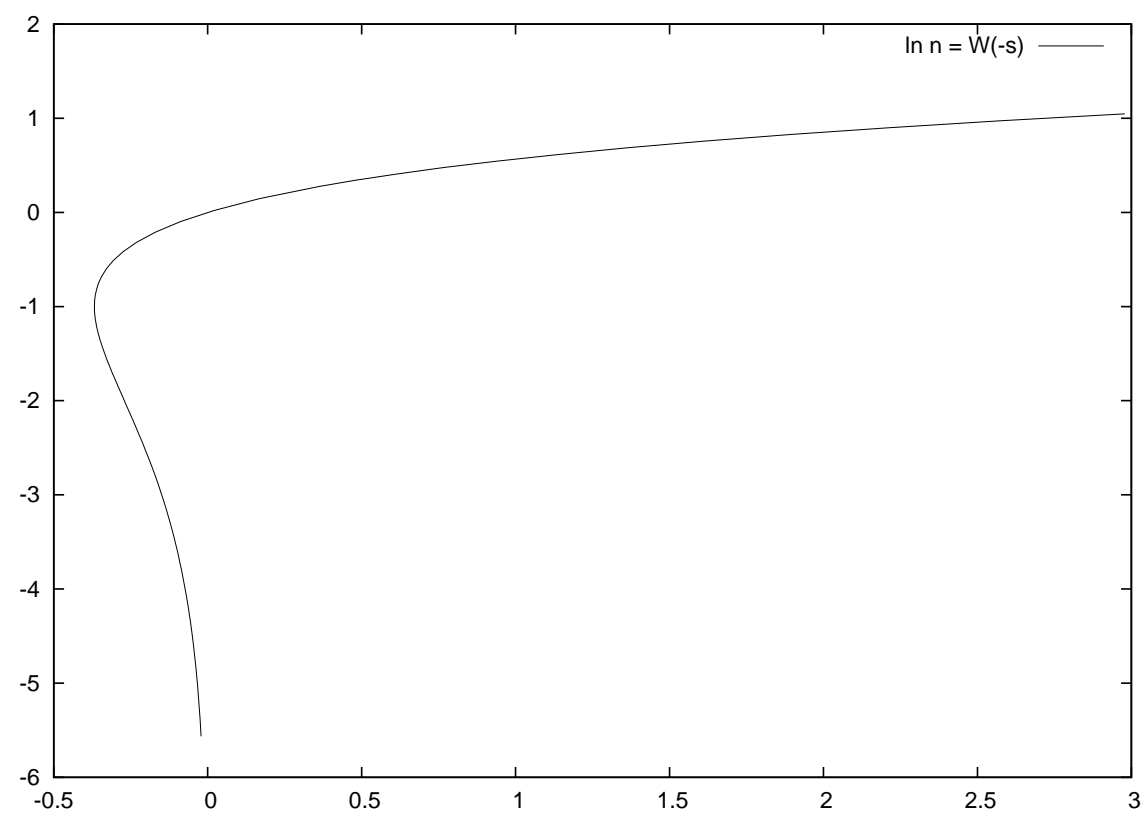

FIG. 3: The logarithm of the density of the Krypton atom as a function of the negative of the local entropy. 